\title{
DIVISION VII
}

\section{THE GALACTIC SYSTEM}

\author{
SYSTÈME GALACTIQUE
}

Division VII provides a forum for astronomers studying the Milky Way as a galactic system, and its constituents.

\author{
PRESIDENT \\ VICE-PRESIDENT \\ PAST PRESIDENT \\ BOARD
}

\author{
Despina Hatzidimitriou \\ Rosemary Wyse \\ Ortwin Gerhard \\ Giovanni Carraro \\ Bruce Gordon Elmegreen \\ Birgitta Nordström
}

\section{DIVISION VII COMMISSIONS}

Commission 33

Structure \& Dynamics of the Galactic System

Commission 37

Star Clusters \& Associations

\section{INTER-DIVISION WORKING GROUPS}

\section{TRIENNIAL REPORT 2009-2012}

\section{Introduction}

Division VII provides a forum for astronomers studying the Milky Way as a galactic system, as well as its constituents. It acts as an umbrella for two commissions, Commission 33 and Commission 37.

Commission 33 focuses on the structure and dynamics of the Galactic System, providing a paradigm for the processes involved in the formation and evolution of the stellar and gaseous components of spiral galaxies in general.

Commission 37 studies star clusters and associations, as test particles for deciphering the formation and evolution of the galactic system as a whole, but also as important astrophysical objects in their own right.

\section{Developments within the past triennium}

\subsection{Observations - Surveys}

There has been a great revival of Milky Way research, in recent years, partly in preparation for the forthcoming GAIA mission. Several large surveys and experiments, either photometric or spectroscopic, in several wavelength regimes, have been recently completed, are in progress, or are planned for the near future: the Sloan Extension for Galactic Understanding and Exploration 2 (SEGUE-2), the RAdial Velocity Experiment (RAVE), the Apache Point Observatory Galactic Evolution Experiment (APOGEE), the HERMES Galactic archaeology survey, the ESO-GAIA spectroscopic survey, the LAMOST Experiment for Galactic Understanding and Evolution (LEGUE), the SkyMapper 
survey, the Young stellar object variability (YSOVAR) survey with Spitzer, the VISTA Variables in The Via Lactea (VVV) public survey, the UKIDSS near infrared galactic plane and galactic clusters survey, the JCMT Legacy Survey (at 450 ìm and 850 ìm), the APEX telescope ATLASGAL 870 micron survey, the Herschel Hi-GAL survey (between 70 and 500 microns), the Caltech sub-millimeter Observatory BGPS Bolocam $1.1 \mathrm{~mm}$ survey, the Spitzer GLIMPSE 360 mid-IR survey, the UKIRT Widefield Infrared Survey for molecular hydrogen, the International Galactic Plane Survey which combines many radio telescope surveys from around the world and the Spitzer and Herschel guaranteed time projects aimed at mapping nearly all of the molecular clouds within 500 pc of the Sun. The results of these surveys combined with the GAIA mission results and the also awaited Large Synoptic Survey Telescope (LSST) contribution are expected to revolutionize our perception of the MW galaxy and our understanding of how galaxies form and evolve, in general.

\subsection{Theory - Simulations}

Simulations, both pure N-body and hydrodynamic, are increasing in resolution and dynamic range and are now capable of following the formation of a Milky Way analogue galaxy within a proper cosmological framework and with fairly realistic modeling of the complex physics of gas dynamics, star formation and chemical evolution. They can now make robust, detailed predictions to be compared with the comprehensive large observational data-sets from the surveys mentioned above. At the same time, simulations have shown that, contrary to assumptions often made, the Milky Way's morphology is strongly affected by even low-mass minor mergers predicted to be common throughout the Universe.

Simulations are also growing in importance in studies of cluster formation and evolution. Complete N-body simulations of globular clusters are now possible, and simulations of star formation in the cluster environment reproduce the stellar initial mass function, mass segregation, hierarchical structure, the formation efficiency and overall time scale.

\subsection{Meetings and publications}

The triennial reports by the organizing committees of Commissions 33 and 37 that are presented in this volume, describe the main developments and activities related to Division VII. Briefly, during the reporting period, there have been over 30 international conferences and workshops either entirely dedicated to or partly related to the galactic system and/or to star clusters and associations. More than 2000 scientific papers have been published in refereed journals, reporting on new observational or theoretical insights into the Galactic System and its constituents (including star clusters and associations).

Inspection of the subjects of the 10 most cited articles (with "Milky Way" in the title) per reporting year (i.e. 30 in all), reveals the focus of the current efforts of the scientific community: Among these 30 papers, almost half are related to the MW dwarf satellites and their role in its evolution. Results from large surveys account for the next largest most cited group of papers, while the rest are related to different MW components (e.g. the nuclear cluster, gaseous clouds, sub-halos, thick disk, spiral arms). A similar analysis of the publications related to star clusters, show that more than a third of the 30 most cited papers (10 per reporting year) are related to the presence of multiple stellar generations in star clusters, while another third study different aspects of metal enrichment in clusters.

\subsection{Business Matters}

The Working Group of Division VII, the "Galactic Center", which was created during the IAU XXVI General Assembly in Prague (2006) has been discontinued during the 
Business Meeting of Division VII at the IAU XXVII General Assembly in Rio Janeiro (2009).

\subsection{Closing Remarks}

The remarkable international effort on large multiwavelength observational surveys both ground based and from space, along with the great advances in theory and simulations, promise that the next decade will see a revolution in our undestanding of the formation and evolution of our Galaxy.

Information on Division VII can be found on the Division web page: http ://www.iau.org/science/scientific_bodies/divisions/VII/. 